\title{
ENHANCEMENT OF THE POWER RADIATED BY A DIPOLE ANTENNA AT BORESIGHT BY MEANS OF A LEFT HANDED SUPERSTRATE
}

\author{
E. Sáenz ${ }^{(1)}$, I. Ederra ${ }^{(1)}$, R. Gonzalo ${ }^{(1)}$, P. de Maagt $^{(2)}$ \\ (1) Electrical and Electronic Engineering Department, Public University of Navarra \\ Campus Arrosadia, E-31006, Pamplona, Navarra, Spain. Tel: +34 948169023 Fax: +34 948169720 \\ E-mail: elena.saenz@unavarra.es,ramon@unavarra.es \\ (2) European Space Research and Technology Centre, ESTEC, \\ PO Box 299, NL 2200 AG Noordwijk, The Netherlands Tel: +31 715655906 Fax: +31 715654999 \\ E-mail: Peter.de.Maagt@esa.int
}

\begin{abstract}
In this paper a comparison between the measured radiation performances of a single dipole and a dipole with a left handed superstrate based on a finite periodic repetition of a unit cell is presented. First of all the return losses and resonant frequency of the dipole has been measured for different sizes of the superstrate, having a good impedance matching and a decrease in the resonant frequency as the number of cells increase. By using an anechoic chamber and a receiver horn antenna, the power transmitted at boresight has been measured for different frequencies, observing a filtering behaviour due to the resonant characteristic of the superstrate and an improvement of the power transmitted at the resonant frequency of around $3 \mathrm{~dB}$. Comparing the $\mathrm{H}$ and $\mathrm{E}$ plane radiation patterns of a dipole and the dipole with superstrate, more symmetrical and directive radiation patterns can be observed. Finally, a comparison between the simulated and measured aperture efficiency is presented with a good agreement.
\end{abstract}

\section{INTRODUCTION}

Nowadays, the study of the so-called metamaterials (MTMs) has been increasing due to the enormous potential of this technology. Examples of MTMs include Photonic Band Gap (PBG) structures and Left Handed Material (LHM). Several recent papers have exposed the usefulness of these MTMs for different applications [1]-[9]. LHMs can be understood as resonators with pass band and stop band properties at which the power is transmitted or reflected respectively. Up to now, volumetric MTMs have been used to create Artificial Magnetic Conductor (AMC) for antenna applications [6]. Working in the rejection bands as substrate of dipole antennas instead of a perfect electric conductor (PEC) ground plane, an enhancement of the directivity and reduction of the back radiation is obtained. Recently, applications of LHMs have shown the benefits of their pass band properties using them as superstrate of planar antennas with the goal of improving their radiation behaviour [5]-[9].

In this paper a comparison between the radiation properties of a single dipole and a dipole with a LH superstrate is presented. Measurements of the $S_{11}$ parameter and the radiation pattern have been carried out for different sizes of the superstrate, i.e., varying the number of unit cells that form the superstrate. Finally, a comparison between the aperture efficiency obtained with these measurements and the one predicted by the simulations with Ansoft-HFSS is shown.

\section{LHM SUPERSTRATE}

The left handed media used as superstrate is a finite periodic structure based on the unit cell described in [3]-[5]. It consists of one Split Ring Resonator (SRR) between two pairs of Capacitively Loaded Strips (CLSs) all embedded in a dielectric slab (see Fig. 1).

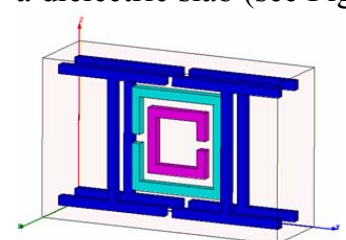

(a)

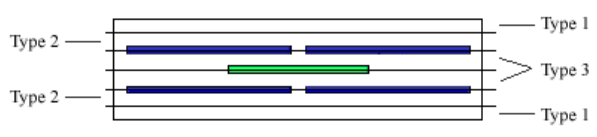

(b)

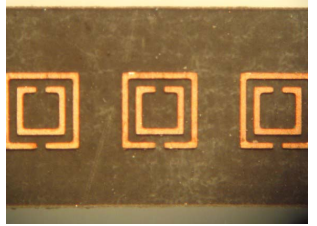

(c)

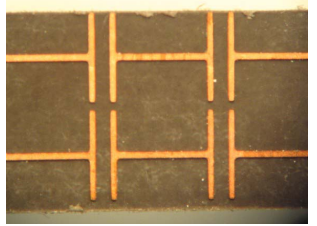

(d)

Fig. 1. (a) Geometry of the LHM unit cell (b) Layers of one period (c) Detail of the SRRs (d) Detail of the CLSs.

In order to construct a unit cell, a layer by layer technique described in detail in [5] is followed. Each period is created by stacking six layers, 2 layers of each of the three types, following the pattern 123321 (see Fig. 1(b)). Using this fabrication technique, the number of periods that constitute the superstrate is not fixed, but can be modified by varying the number of layers stacked. 


\section{RADIATION PERFORMANCES}

In order to check the improvements in the radiation performances of a dipole when a LH superstrate is used, the $\mathrm{S}_{11}$ parameter and the radiation pattern have been measured and compared with the case of a single dipole.

To excite correctly the superstrate, an E field parallel to the CLSs and an H field axial to the SRRs are required. To do so, a $\lambda / 2$ dipole fed by a coaxial balun was designed and used as feeding point.

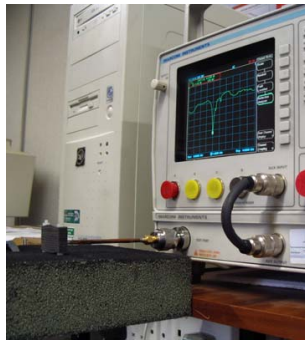

(a)

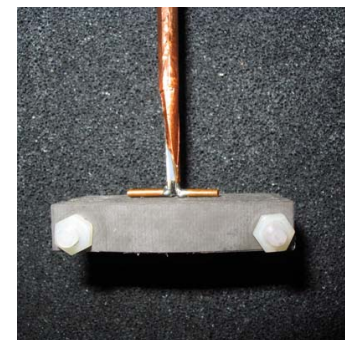

(b)

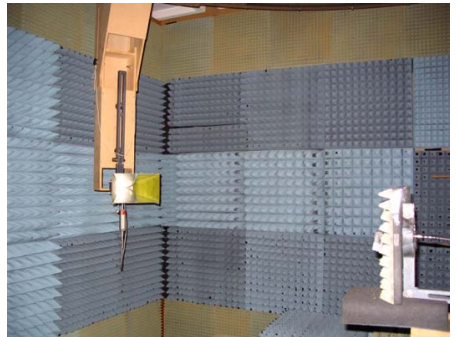

(c)

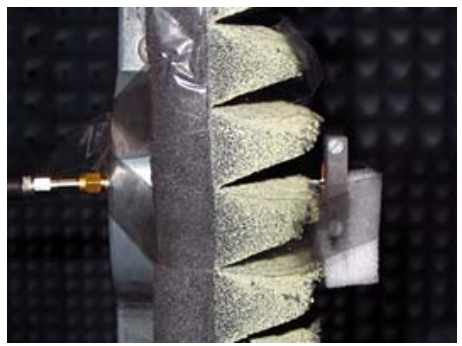

(d)

Fig. 2. (a) (b) Set-up for the measurements of the $S_{11}$ parameter. (c) (d) Set-up for the measurements of the radiation patterns.

\section{A. $S_{11}$ Parameter}

First of all, the $S_{11}$ parameter of the dipole with the superstrate was measured by using a network analyser. The set up of the measurements is shown in Fig. 2 (a) and (b).

The influence of the LHM superstrate in the impedance matching performances of the dipole was analyzed by varying the number of periods of the superstrate from 2 to 12 . The magnitude of the $S_{11}$ parameter vs the frequency for all the different configurations measured is shown in Fig. 3 (a). A matching better than $-12 \mathrm{~dB}$ has been obtained for all the cases.

Fig. 3 (b) shows the evolution of the resonant frequency of the configuration (LHM + dipole) as the number of cells that form the superstrate increases. Three types of resonances has been taken into account, the frequency of the minimum $\mathrm{S}_{11}$, the frequency of the maximum gain measured at boresight in the $\mathrm{E}$ plane and the frequency of the maximum gain in the $\mathrm{H}$ plane. The method followed to measure the $\mathrm{H}$ and $\mathrm{E}$ radiation patterns will be explained in the following section B. A similar behaviour can be observed in all cases apart from a little shift due to small variations in the relative position of the superstrate with the dipole when the measurements were carried out. It can be observed that as the number of cells increases, the resonant frequency decreases varying from $11.36 \mathrm{GHz}$ in the case of 2 cells to $10.96 \mathrm{GHz}$ in the case of 12 cells.

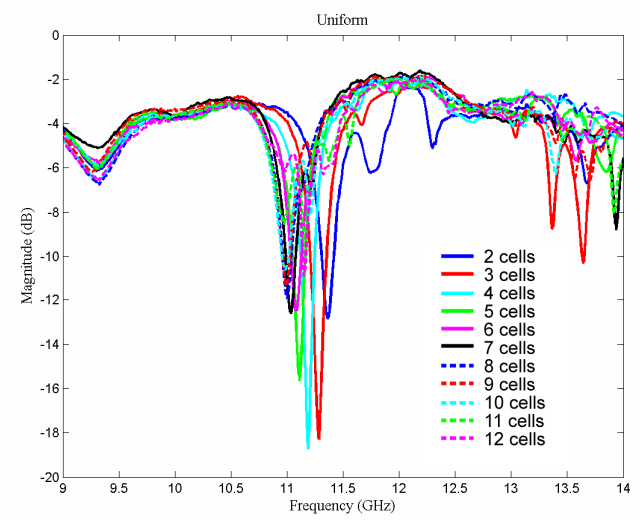

(a)

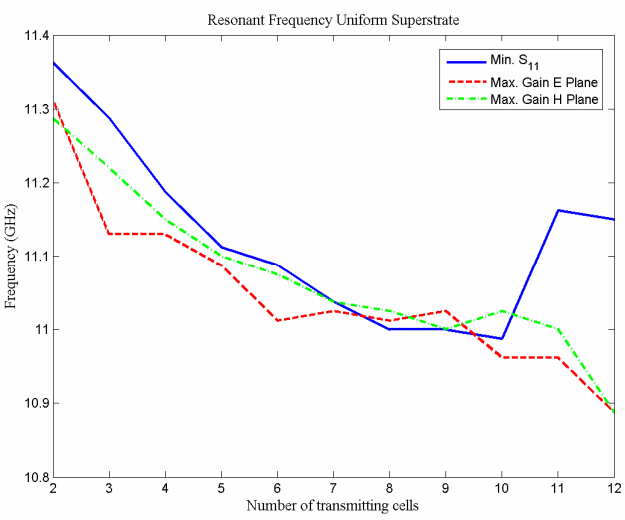

(b)

Fig. 3. (a) Magnitude of the $S_{11}$ vs frequency. (b) Resonant frequency vs number of cells. 


\section{B. Radiation Pattern}

The next step in the characterization of the enhancement of the radiation performances due to the use of the superstrate was the measurement of the $\mathrm{E}$ and $\mathrm{H}$ plane radiation patterns. To do so, a half anechoic chamber with a horn antenna as receiver and the dipole (with and without superstrate) as transmitter were used. The position of the superstrate was selected in order to maximize the power received at boresight, i.e., the position of maximum gain. Some pictures of the set-up of the measurements are shown in Fig. 2 (c) and (d).

Analysing the power received vs the frequency (see Fig. 4 (a)), a filtering behaviour can be observed due to the pass band properties of the superstrate. Comparing this power received when the dipole has the superstrate with the case of the single dipole, an enhancement around the resonant frequency of the superstrate can be observed. Out of this band, a rejection of around $-10 \mathrm{~dB}$ has been obtained.

Measuring the radiation pattern (power received $P_{R}$ ) at that resonant frequency and taking into account the power transmitted $P_{T}$, the gain of the receiver horn antenna $G_{R}$, the distance between antennas $d$ and the working frequency $\lambda$, the gain of the transmitting antenna $G_{T}$ can be calculated by applying the Friis equation (1).

$$
G_{T}=20 \log \left(\frac{4 \pi d}{\lambda}\right)+P_{R}-P_{T}-G_{R}
$$

As in the case of the $S_{11}$, a parametric analysis has been carried out varying the number of cells from 2 to 12 . Fig. 4 shows the results obtained in the case of a superstrate with 9 periods. Looking at the frequency sweep of the power received (see Fig. 4 (a)), an enhancement of $3 \mathrm{~dB}$ at the resonant frequency of $11 \mathrm{GHz}$ can be observed. Comparing the radiation patterns with and without superstrate at that frequency (see Fig. 4 (b)), it can be seen that the $\mathrm{H}$ and E planes for the superstrate case are more symmetrical and directive.

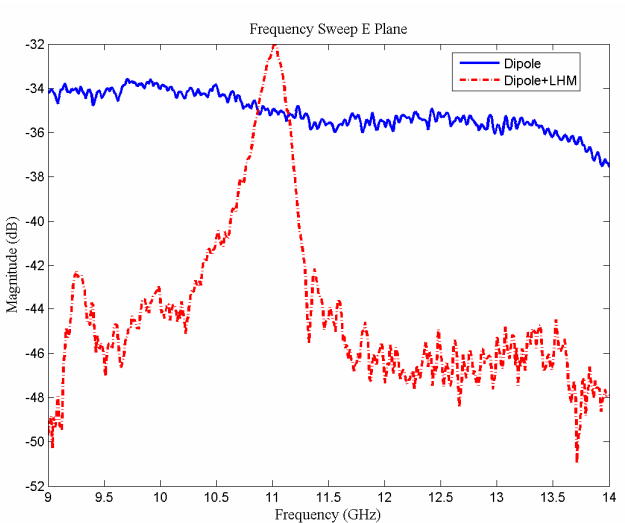

(a)

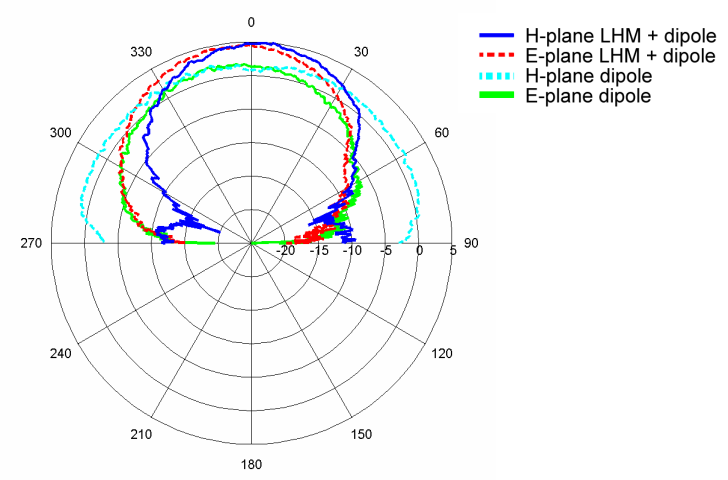

(b)

Fig. 4. (a) Power received vs frequency (b) $\mathrm{H}$ and $\mathrm{E}$ plane radiation pattern.

\section{Aperture Efficiency}

Once the gain $G$ of the configuration is known, the aperture efficiency $(\eta)$ of the configuration can be calculated following (2)

$$
G=\frac{4 \pi}{\lambda^{2}} \eta A_{p h y s}
$$

where $A_{\text {phys }}$ is the physical area of the superstrate.

A comparison between the aperture efficiency achieved with the measurements and the one of the simulations for the different number of cells analysed can be seen in Fig. 5. A good agreement between them can be observed. 


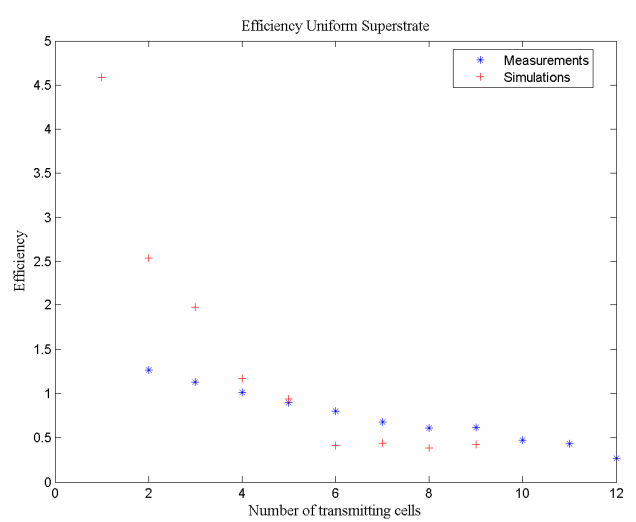

Fig. 5. Aperture efficiency vs the number of transmitting cells.

Due to the small dimensions of the superstrates, there is field on the edges of the superstrate so the radiation area is higher than the physical area. As a result, the aperture efficiency is larger than one. It can be observed that as the number of cells increases, the efficiency decreases. Although the gain increases with the number of cells, the ratio of increasing surface is higher, so the efficiency decreases. Besides, as the physical area increases, the edge effect decreases, achieving an expected result of an aperture efficiency smaller than one, but higher than $80 \%$ until six cells.

\section{CONCLUSIONS}

In this paper the radiation performances of a dipole and a dipole with a LH superstrate are compared. A parametric analysis of the influence of the superstrate in the radiation performances of the configuration has been carried out varying the number of periods of the superstrate from 2 to 12 . In all cases, a good impedance matching was obtained with a $\mathrm{S}_{11}$ parameter smaller than $-12 \mathrm{~dB}$ at the resonant frequency. It was observed that when the number of periods increases, the resonant frequency decreases. Measuring the power transmitted at boresight by the dipole with superstrate and comparing with the one radiated by the single dipole, an enhancement of around $3 \mathrm{~dB}$ was achieved around the resonant frequency of the superstrate. Comparing the $\mathrm{H}$ and $\mathrm{E}$ plane radiation patterns at that frequency, more symmetrical and directive radiation patterns can be observed with this new configuration. Finally, it has been observed that the aperture efficiency decreases with the number of cells having an aperture efficiency larger than $80 \%$ until 6 cells.

\section{REFERENCES}

[1] V.G. Veselago, "The electrodynamics of substances with simultaneously negative values of $\varepsilon$ and $\mu$ ", Sov. Phys. Uspekhi, 1968, Vol. 10, No 4, pp. 509-514.

[2] J. B. Pendry, "Negative refraction makes a perfect lens", Physical Review. Lett, 2000, Vol. 85, No 18, pp. 39663969.

[3] R. W. Ziolkowski, "Design, fabrication, and testing of double negative metamaterials", IEEE Trans. Antennas and Prop., 2003, Vol. 52, No 7, pp. 1516-1529.

[4] E. Sáenz, R. Gonzalo, I. Ederra, P. de Maagt, "High efficient dipole antennas by using left-handed superstrates", Proc. $13^{\text {th }}$ International Symposium on Antennas JINA 2004.

[5] E. Sáenz, R. Gonzalo, I. Ederra, P. de Maagt, "Transmission enhancement between rectangular waveguides by means of a left handed media", Electronics Letters, 2005, Vol. 41, No 13, pp. 725-727

[6] A. Erentok, P. L. Luljak, R. W. Ziolkowski, "Characterization of a volumetric metamaterial realization of an artificial magnetic conductor for antenna applications" IEEE Tran. Antennas and Prop, 2005, Vol. 53, No. 1, pp. 160-172.

[7] Young Ju Lee, Junho Yeo, Raj Mittra, Wee Sang Park, "Design of a high-directivity electromagnetic band gap (EBG) resonator antenna using a frequency-selective surface (FSS) superstrate", Microwave and Optical Technology Lett, 2004, Vol. 42, No 6, pp. 462-467.

[8] Young Ju Lee, Junho Yeo, Raj Mittra, Wee Sang Park, "Application of electromagnetic bandgap (EBG) superstrates with controllable defects for a class of patch antennas as spatial angular filters", IEEE Trans. Antennas and Prop, 2005, Vol. 53 No. 1, pp. 224-235.

[9] Pekka Ikonen, Constantin Simovski, Sergei Tretyakov, "Compact directive antennas with a wire-medium artificial lens”, Microwave and Optical Technology Lett, 2004, Vol. 43, No. 6, pp. 467-469. 